\title{
Renal Tuberculosis in a 14-Years-Old Boy Presented as Pyrexia of an Unknown Origin (PUO): A First Case Report
}

\author{
Sam Hassan ${ }^{1, *}$, Ali Hassan Abro ${ }^{2}$ \\ ${ }^{1}$ Department of Paediatrics, Mediclinic City Hospital, Dubai, United Arab Emirates \\ ${ }^{2}$ Infectious Diseases Unit, Rashid Hospital, Dubai, United Arab Emirates
}

Email address:

samhassan@gmail.com (S. Hassan), bahabro@dha.gov.ae (A. H. Abro)

${ }^{*}$ Corresponding author

To cite this article:

Sam Hassan, Ali Hassan Abro. Renal Tuberculosis in a 14-Years-Old Boy Presented as Pyrexia of an Unknown Origin (PUO): A First Case Report. American Journal of Pediatrics. Vol. 4, No. 2, 2018, pp. 21-24. doi: 10.11648/j.ajp.20180402.11

Received: March 11, 2018; Accepted: April 9, 2018; Published: May 14, 2018

\begin{abstract}
Tuberculosis remains an important public health problem, especially in developing countries. Mycobacterium Tuberculosis mainly affects the lungs but extra-pulmonary Tuberculosis is not uncommon. However, renal involvement is very rare particularly in paediatric age group. This article is reporting a case of 14 years old Emirati boy who presented with history of fever, mild cough and night sweats after admission. The patient was treated as a case of fever of unknown origin, most likely secondary to community acquired pneumonia. On further investigations, he was found to have pulmonary tuberculosis with miliary pattern of the both lungs and also renal tuberculosis as the urine culture grew Acid Fast Bacilli (AFB) (Mycobacterium Tuberculosis). The patient responded very well to the Anti-tuberculosis treatment and became symptom free.
\end{abstract}

Keywords: Mycobacterium, Renal Tuberculosis, Pulmonary Tuberculosis, PUO

\section{Introduction}

Tuberculosis is caused by Mycobacterium Tuberculosis and the lungs are infected most commonly, whereas extrapulmonary tuberculosis accounts only $22 \%$ cases of tuberculosis. The incidence of Pulmonary and Extrapulmonary tuberculosis has been increased recently mainly due to increasing prevalence of HIV and Diabetes Mellitus as well as common use of Immunosuppressive drugs [1]. According to WHO world TB report 2014, there were an annual incidence of 9 million cases of TB. One and half million People die of the disease every year [2]. In the developed countries the urogenital tuberculosis accounts for $27 \%$ of extrapulmonary TB. It is the third most frequent form of extrapulmonary TB after pleural and lymphatic TB. It spreads by hematologic dissemination of the pulmonary tuberculosis in almost all cases. Whereas, it's incidence is quite low in the developing countries, most likely due to under diagnosis because of unavailability of diagnostic facilities [3]. Renal involvement is of great significance, especially in children because in comparison to adults the incidence is quite low in paediatric age group [4].
Furthermore, due to the non-specific presentation of renal tuberculosis, diagnosis often delayed leading to increased morbidity and mortality. Renal tuberculosis is either late reactivation or complication of pulmonary tuberculosis and only a few cases reported in literature with miliary tuberculosis [5]. This paper, is to report a 14 years old boy presented with pyrexia of unknown origin (PUO) for investigation and found to have renal tuberculosis associated with miliary tuberculosis. Searching the English literatures none were found about renal $\mathrm{TB}$ in children presented as PUO before. This case signifies the importance of the CT scan imagining and the urinary culture for AFB when there is no other explanation for the PUO. It also denotes the importance of the early diagnosis and treatment of the GUTB before any serious and detrimental renal complications develop.

\section{Case Report}

A 14 years old boy, not known to have any previous medical illness. He was fully immunized including the BCG vaccine which was given after birth. He was admitted with the complaints of fever and mild dry cough for 15 days. For 
the last few days before admission he had mild abdominal discomfort on-and-off with nausea and vomited once. There was no change in the appetite and weight. The patient denied any urinary or bowel complains. Multiple antibiotics were given from outside clinics including Macrolide and Penicillin before presenting to the hospital. On clinical examination, the developmental milestones were within reference range according to the age group. The patient looks pale, however all the other systemic examinations were normal. The initial investigations showed normal white blood cell (WBC) count with differential in reference range. Platelets were normal but haemoglobin was $10.8 \mathrm{gm} / \mathrm{dl}$ with normochromic normocytic RBCs. The liver function test (LFT), blood sugar and urea and electrolytes were normal. The ESR was $56 \mathrm{mmHg} / 1 \mathrm{~h}$, CRP $51 \mathrm{mg} / \mathrm{dl}$, and blood culture was negative. The chest Xray (Figure 1) showed a few infiltrates mainly on the left side in the middle zone. The mid-stream urine (MSU) analysis showed protein $1+$, WBC $25-30$ cell/uml and RBC $40-45$ cells/uml but urine culture was negative.

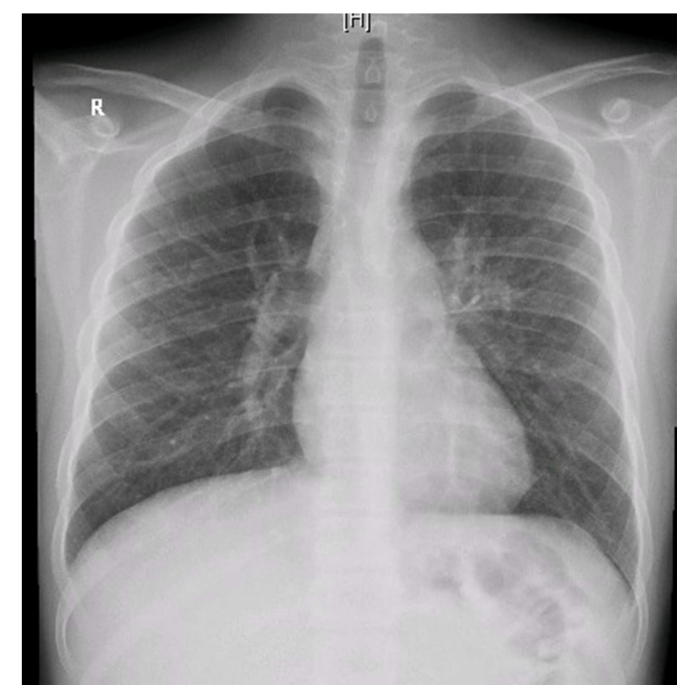

Figure 1. $X$-ray chest PA view shows infiltrate in left lung.

Considering the clinical history and the laboratory results, community acquired pneumonia was considered as the most likely cause of the patient's fever. Hence, the patient was started on Ceftrixone and Azithromax. After three days of treatment, the patient remained febrile and antibiotic coverage was escalated to Meropenem and Vancomycin. The respiratory screening for viruses and Mycoplasma screening turn out to be negative. On further inquiry, it was revealed by the patient's mother that the grandfather and aunty had suffered from pulmonary tuberculosis and were treated accordingly. After four days of new antibiotics the patient remained febrile. The connective tissue screening was found to be negative as well as the echocardiogram was normal but the ESR rose to $74 \mathrm{mmHg} / 1^{\text {st }}$ hour, CRP $81 \mathrm{mg} / \mathrm{dl}$ and had persistent proteinuria, pyuria and haematuria. Meanwhile Mantoux Test (Tuberculin test) was order and its reading was $15 \mathrm{~mm}$ (Figure 2) after 72 hours.

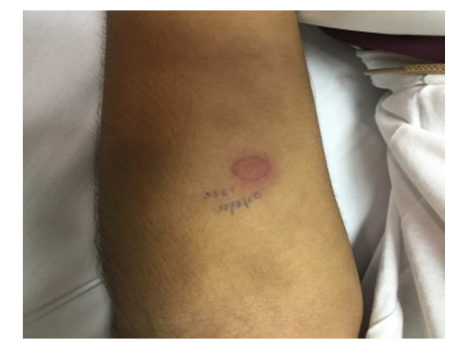

Figure 2. Mantoux Test (Tuberculin test)

Due to non-response to antibiotics and positive MT, antibiotics were stopped and CT scan chest, T-spot TB test and urine for AFB culture were requested. The CT scan chest (Figure $3 \mathrm{~A}$ ) showed pattern of miliary tuberculosis and urine culture also grown AFB. However the T-Spot TB test initially was indeterminate while the repeated test after one week was negative. The ultrasound scan of the kidneys reported normal but the CT abdomen (Figure 4) showed low density areas in both kidneys. Sputum for AFB could not be done as he was not producing sputum and gastric lavage could not be performed because family did not agree on it.

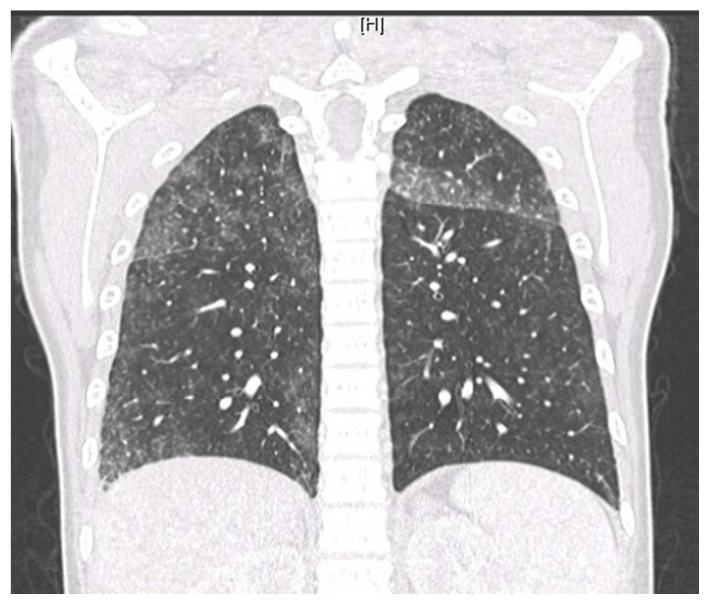

A

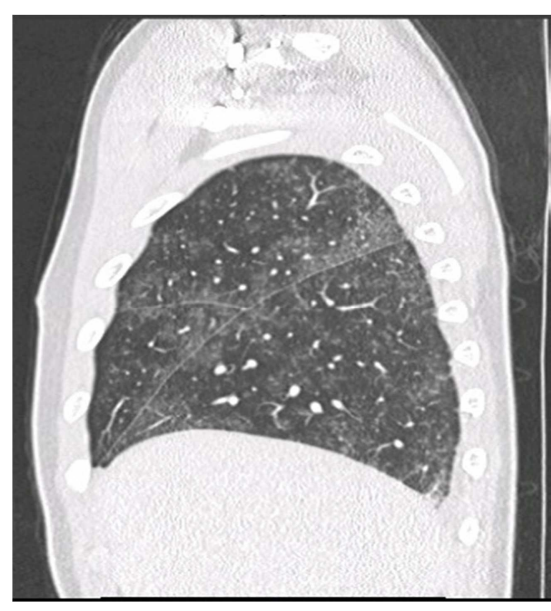

B

Figure 3. $A \& B$ show miliary pattern with some ground glass appearance bilaterally. 


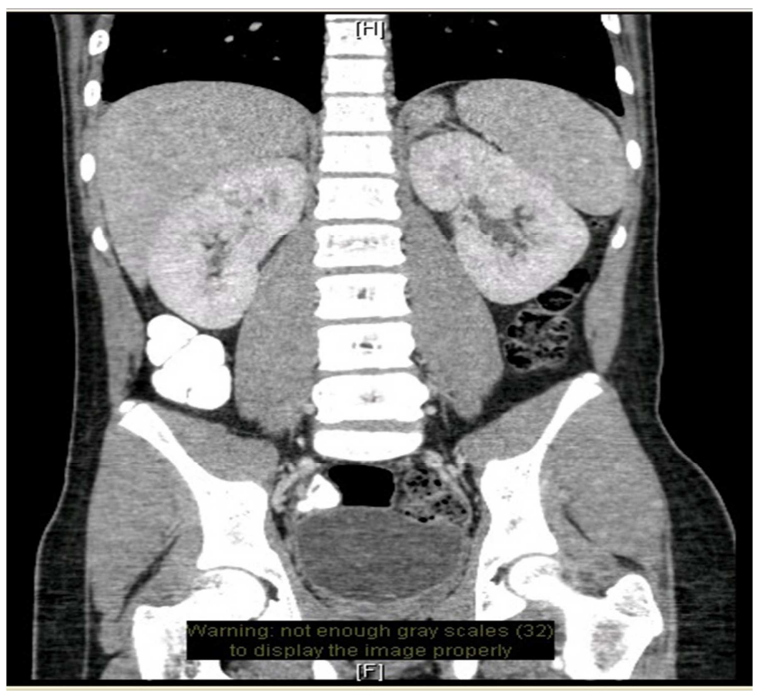

Figure 4. T scan of kidneys shows bilateral hypo-densities.

After receiving the results of $\mathrm{CT}$ scan chest, MT and urine for AFB culture, the patient was labelled as a case of Pulmonary Tuberculosis with renal involvement. The patient was started on four drugs Rifampcin, Isoniazid, Pyrazinamide and Ethambutol (RHZE) regimen, and responded very well to ATT and became afebrile after 7 days of the treatment and was discharged from the hospital with out-patients follow. On follow-ups he remained symptoms free and the repeat urine AFB became negative after 1 month of the treatment. In total, he received six months of completed treatment and was discharged from the clinic with symptoms free.

\section{Discussion}

Renal tuberculosis is invariably secondary to distant (disseminated) foci of infection, most notably from lungs. Primary (localized) infection of kidney is rare. In both conditions (disseminated and localized) the intensity of infection depends upon the infecting dose, virulence of the organism and the resistance of the host [6]. In miliary TB (disseminated form) most tubercles are located in the cortex leading to formation of granuloma with giant cells and renal function is abnormal, likely due to interstitial nephritis. In localized renal disease, the origin of bacilli is pulmonary but lung disease is rarely found, however there might be clinical or radiological traces of lung disease $[5,7]$. In urogenital tuberculosis (disseminated or localized), typical lesions (granuloma) start in the kidneys and spread distally to ureters, bladder and testicles. Renal disease may present with proteinuria, pyuria, haematuria and loss of kidney function. Symptoms suggestive of urinary tract infection with pyuria and haematuria but negative routine urine culture are similarly suggestive of urogenital tuberculosis. In advance disease it may cause obstructive uropathy, bladder defect and loss of kidney function leading to chronic renal failure. Diagnostic imaging may show calcification, distorted ureters with alternate stenosis and dilatation, reduced bladder volume, hydro-nephrosis, chalice distortion, cavities and decreased kidneys size and function in advance disease [8, 9].

Genitourinary tuberculosis (GUTB) commonly present at adult age as it takes 5-20 years after infection to present clinically. The prevalence of GUTB in paediatric age group is quite low and it accounts less than $5 \%$ of extrapulmonary tuberculosis compare to $27 \%$ in adult population [3 and 10]. The symptoms and signs of genitourinary tuberculosis are often vague and insidious and represent a challenge for clinicians, especially for paediatricians due to rare presentation of the disease. Isolated renal TB is rarely symptomatic presenting with proteinuria, pyuria, and haematuria with repeated bacteriological culture negative and progressive unexplained decline in renal function. Dysuria, nocturia, frequency and urgency of micturition predominate only when ureters or lower urinary tract is involved and even these symptoms are less noticeable in children [11]. The hallmark of renal TB is sterile pyuria followed by microscopic painless haematuria, which is seen in $50 \%$ of cases. Fever, weight loss and night sweat are too unusual in renal tuberculosis. Only one-third of cases of the renal tuberculosis have an abnormal chest X-ray [5]. In this case, the positive clinical parameters were fever, night sweat, sterile pyuria and AFB grown in the urine, however the patient was initially presented as pyrexia of unknown origin (PUO). It is the first report in a child to present as PUO due to renal TB.

Renal tuberculosis occurs concomitantly with active pulmonary tuberculosis in $40 \%$ of cases. In this patient, respiratory symptoms, pulmonary infiltrates on the chest Xray and miliary pattern on $\mathrm{CT}$ scan chest supported the diagnosis of pulmonary tuberculosis concomitant with renal tuberculosis. Furthermore, according to several reports, in case of renal involvement during pulmonary tuberculosis, urine smear for AFB will be positive in up to $50 \%$ on repeated analysis and culture will be positive in $80-90 \%$ [12, 13]. In this case, AFB was isolated in the urine which supports the above reports that $\mathrm{ABF}$ can be isolated in the urine in the renal tuberculosis cases.

Genitourinary tuberculosis generally responds better to medical treatment than pulmonary tuberculosis because of lower Mycobacterial load and better penetration of antituberculosis drugs into cavitatory lesions of kidneys and maintenance of high concentration of drugs in the urine. Four-drug regimen (RHZE) for six months is quite effective in eradicating the renal tuberculosis and it is preferable to give directly observed therapy. All the four drugs should be given for two months (initial phase) followed by Rifampicin and Isoniazid for further 4 months (continuation phase). The role of steroid is not proven to be of much benefit, whereas various surgical procedures have important role in the management of renal tuberculosis in certain situation such as when there is obstruction, pyonephrosis, sever calcification and patient is not responding to ATT and non-functioning kidney [11]. The urinary tuberculosis is a great mimicker hence a high index of suspicion is necessary to make a correct and timely diagnosis of 
GUTB [14].

\section{Conclusion}

In conclusion, renal tuberculosis is a quite uncommon in paediatric age group and it is underdiagnosed because of its nonspecific presentation and unavailability of diagnostic facilities, especially in developing countries. Paediatricians should keep a low threshold for suspecting renal tuberculosis, particularly when patient presenting with pyuria and haematuria plus repeated negative bacteriological cultures of urine. Moreover, early diagnosis can prevent significant disease process, which can lead to renal insufficiency, obstruction and end stage renal disease (ESRD). Cases presented as PUO with no cause identified, TB should be considered. Direct questioning about detailed family history of previous TB is very important as in some cultures families may consider TB as stigma and hide it. In this case family history of TB was initially withheld until direct questioning and mother later volunteered to disclose it. Carrying out appropriate urine, radiological and skin tests are highly important to have timely diagnosis.

\section{References}

[1] Rieder HL, Snider DE Jr, Cauthen GM. Extrapulmonary tuberculosis in the United States. Am Rev Respir Dis 2009; 141: 347-351.

[2] World Health Organisation (WHO) global tuberculosis report 2014.

[3] Elizabeth DFD, Geraldo BdSJ and Elvino JGB. Review; Renal Tuberculosis in the Modern Era. Am. J. Trop. Med. 2013; 88(1):54-64.

[4] Arora N, Saha A and Kaur M; Tuberculosis pyelonephritis in children: three case reports; J Paediatrics and International Child Health; 2017; 37 (4): 292-297.
[5] Estwood JB, Corbishley CM, Grange JM. Tuberculosis and the Kidney. J Am Doc Nephrol 2001; 12: 1307-1314.

[6] Muttarak M, ChiangMai WN, Loranapiwat B. Tuberculosis of the genitourinary tract: Imaging features with pathological correlation. Singapore Med J 2005; 46: 568-574.

[7] Yadav S, Singh P, Hemal A, and Kumar R; Gental tuberculosis: current status of diagnosis and management; Transl Androl Urol; 2017: 6(2): 222-233; doi: 10.21037/tau.2016.12.04.

[8] Gambhir S, Ravina M, Rangan K, Dixti M, Barai S, Bomanji $\mathrm{J}$; Imaging in extrapulmonary tuberculosis; International $\mathrm{J}$ of infectious diseases; 2017; 56: $237-247$.

[9] [9] Figueirdo AA, Lucon AM, Arvellos AN, Ramos CO, Toledo AC, Falci R Jr, Gomes CM, Recaverren FE, Netto JM, Srougi M. A better understanding of urogenital tuberculosis pathophysiology based on radiological findings. Eur J Radiol 2010; 76: 246-257.

[10] Dodd JP, Yuen C M; The global burden of tuberculosis mortality in children: a mathematical modelling study; Lancet Glob Health, 2017; 5(9): e898-e906; doi: 10.1016/S2214109X(17)30289-9

[11] Ruhil K, Saif Q, Arun V. Renal Tuberculosis: The Engima Continues. Mycobact Dis 2015; 5(3): 186-192.

[12] Rom WN, Garay S. Tuberculosis. W. B Sauders, USA. 2000; 47: 609-16.

[13] Matsumura N, Yamamoto K, Hirohashi R, Kitano S. Renal tuberculosis mimicking hydronephrosis. Intern Med 2005; 44 (7): 768 .

[14] Krishnamorthy s, Pakaniyandi V, Kumaresan N, Govindaraju S, Rajasekaran J, Murugappan I, Ramanan V, and Krishnan M $\mathrm{N}$; Aspects of Evolving Genito Urinary Tuberculosis-A Profile of Genito Urinary Tuberculosis (GUTB) in 110 Patients; J Clin Diagn Res. 2017; 11(9): PC01-PC05.

[15] Published online 2017. doi:10.7860/JCDR/2017/25882.10557. 